\title{
A Middle Age Woman with the Episodes of Excruciating Pain in the Neck and Ear
}

\author{
Ashfaque Memon", Adil Samad and Katherine Finan
}

General Medicine, Sligo University Hospital, County Sligo, Ireland

"Corresponding Author: Dr. Ashfaque Memon, General Medicine, Sligo University Hospital, County Sligo, Ireland, Tel: 0851464517; E-mail: dr_agha80@hotmail.com

Received: 15 March 2018; Accepted: 04 April 2018; Published: 01 May 2018

\begin{abstract}
A middle aged woman presented with episodes of excruciating pain in right side of neck and right ear. The pain was episodic and each episode lasting for few seconds. Multiple doses of NSAIDs, steroids and morphine had no effect over the intensity and frequency of pain. MRI brain, neck and internal auditory meatus were normal. She was reviewed by ENT and dental services and no obvious cause was detected. A clinical diagnosis of Glossopharyngeal neuralgia was made and escalating dose of carbamazepine was started which brought remarkable pain control.
\end{abstract}

Keywords: Glossopharyngeal neuralgia; Neuralgic pain; Neck and ear pain

\section{Introduction}

Glossopharyngeal neuralgia is a relatively uncommon neuralgic pain condition. More importantly it often remains unrecognised due to the lack of awareness about this disorder. This case report could lead medical professionals to consider GPN as a differential diagnosis when dealing with such atypical pain in face, neck and ear.

\section{Case report}

A 50 years old lady woke up with stabbing sharp pain around the back of right ear which lasted for few minutes. Subsequently she had multiple episodes of pain of shorter durations lasting for few seconds, recurring frequently and precipitated by swallowing and drinking hot or cold drinks. The pattern of the each episode was consistent; arising from back of throat, radiating to the external aspect of right ear and mastoid. There was no associated history of headache, fever, ear discharge, toothache, cough or any other related symptom. She had no relevant past medical or 
family history. She is mother of two children, working in regional disability centre. She had normal social life, a non-smoker with no significant alcohol history. On examination she looked extremely anxious and frightened, but otherwise afebrile and haemodynamically stable. There was no tenderness, redness and swelling in the neck, ear and mastoid area. ENT and dental review couldn't detect any gross pathology. She received Antibiotics, steroids, NSAIDs and morphine, with no relief in pain. Laboratory and radiological work up including ESR, CRP, MRI Head, neck and Internal Auditory meatus were normal.

After excluding infective, structural and inflammatory causes a clinical diagnosis of Glossopharyngeal neuralgia was made. She was started on carbamazepine, which effectively reduced the pain episodes. All other analgesics were stopped and carbamazepine dose was escalated till she became completely pain free.

\section{Discussion}

Glossopharyngeal neuralgia (GPN) is a rare or perhaps a rarely diagnosed neuropathic condition, characterized by the episodes of excruciating pain in throat, neck and ear, usually triggered by sweet, acid, cold or hot food and drinks. These episodes are typically of short duration and occur in the distribution of glossopharyngeal nerve [1].

A sub classification of GPN is introduced by the international headache society which divides GPN into classic and symptomatic forms. In classic type, pain occurs intermittently and patient is typically pain free between episodes. Most cases of classic type GPN are idiopathic with no underlying aetiology or associated neurological deficit. In contrast, symptomatic type is usually associated with the underlying structural lesion, which results in persistent discomfort between the episodes of severe neuralgic pain and sensory impairment in the distribution of glossopharyngeal nerve [2].

Though overall GPN is less common than trigeminal neuralgia; but probably not as uncommon as reported, it is more likely that it remains undiagnosed due to overlap with other disorders causing similar paroxysms of pain, such as trigeminal neuralgia and styalgia [1]. The overall reported incidence of GPN is very low (0.2 and 0.7 per 100,000 people per year), but again this incidence is probably underestimated due to insufficient awareness of this rare neurologic condition [3, 4]. Nearly $10 \%$ of the patients with GPN may develop bradycardia, confusion, syncope or convulsions due to the pain distribution along the vagal sensory pathway and is termed as vagoglossopharyngeal neuralgia [5,6]. The diagnosis of GPN is clinical as no standard radiological or laboratory test could be reliably linked with the diagnosis of this disorder. MRI of head and neck is advised to exclude any structural lesion. High resolution MRI with 3D constructive interference in steady state (CISS) can show neurovascular compression of glossopharyngeal or adjacent nerves [7].

First line management of GPN is pharmacotherapy. Membrane stabilizers such as carbamazepine, gabapentin, and pregabalin are first line drugs [8]. NSAIDs are not much helpful for treating such neuralgic pain [9]. Opioids though not very effective for pain but can relieve anxiety [10]. Glossopharyngeal nerve block is an excellent adjuvant to the pharmacotherapy in the management of GPN. Surgical treatment is an option for cases refractory to medications and nerve block; however surgery is associated with high morbidity. 


\section{References}

1. Olds MJ, Woods CI, Winfield JA. Microvascular decompression in glossopharyngeal neuralgia. Am J Otol 16 (1995): 326-330.

2. Headache Classification Subcommittee of the International Headache Society: The International Classification of Headache Disorders: 2nd edition. Cephalalgia 24 Suppl 1 (2004): 9-160.

3. Katusic S, Williams DB, Beard CM, et al. Incidence and clinical features of glossopharyngeal neuralgia, Rochester, Minnesota, 1945-1984. Neuroepidemiology 10 (1991): 266-275.

4. Koopman JSHA, Dieleman JP, Huygen FJ, et al. Incidence of facial pain in the general population. Pain 147 (2009):122-127.

5. Jacobson RR, Russell RW. Glossopharyngeal neuralgia with cardiac arrhythmia: a rare but treatable cause of syncope. Br Med J 1 (1979): 379-380.

6. Kong Y, Heyman A, Entman ML, et al. Glossopharyngeal neuralgia associated with bradycardia, syncope, and seizures. Circulation 30 (1964): 109-113.

7. Karibe H, Shirane R, Yoshimoto T. Preoperative visualization of microvascular compression of cranial nerve IX using constructive interference in steady state magnetic resonance imaging in glossopharyngeal neuralgia. J Clin Neurosci 11(2004): 679-681.

8. Dworkin $\mathrm{RH}, \mathrm{O}^{\prime}$ Connor $\mathrm{AB}$, Backonja $\mathrm{M}$, et al. Pharmacologic management of neuropathic pain: Evidence-based recommendations. Pain 132 (2007): 237-251.

9. Yeng LT. Pharmacological treatment of neuropathic pain. Drugs Today (Barc) 45 (Suppl C) (2009): 7-12.

10. Yang M, Zhou M, He L, et al. Non-antiepileptic drugs for trigeminal neuralgia. Cochrane Database Syst $\operatorname{Rev}$ (2011): CD004029.

Citation: Ashfaque Memon, Adil Samad, Katherine Finan. A Middle Age Woman with the Episodes of Excruciating Pain in the Neck and Ear. Archives of Clinical and Medical Case Reports 2 (2018): 65-67. 